\title{
Propriospinal Bypass of the Serotonergic System That Can Facilitate Stepping
}

\author{
Yury Gerasimenko, ${ }^{1,2}$ Pavel Musienko, ${ }^{1}$ Irina Bogacheva, ${ }^{1}$ Tatiana Moshonkina, ${ }^{1}$ Alexandr Savochin, ${ }^{1}$ Igor Lavrov, ${ }^{2}$ \\ Roland R. Roy, ${ }^{2,3}$ and V. Reggie Edgerton ${ }^{2,3,4}$ \\ ${ }^{1}$ Pavlov Institute of Physiology, St. Petersburg 199034, Russia, and Departments of ${ }^{2}$ Physiological Science and ${ }^{3}$ Neurobiology and ${ }^{4}$ Brain Research Institute, \\ University of California, Los Angeles, Los Angeles, California 90095
}

The neurotransmitter systems mediating spinal locomotion in response to epidural spinal cord stimulation (ES) have not been identified. Here, we examine the role of the serotonergic system in regulating locomotor behavior of decerebrated cats during ES at L4-L5. ES elicited coordinated, weight-bearing, hindlimb stepping with plantar foot placement on a moving treadmill belt. Ketanserin [a 5-hydroxytryptamine (serotonin) (5-HT) $2 / 7$ receptor antagonist] depressed this locomotor activity: only weak rhythmic movements without plantar foot placement and depressed EMG activity were observed. Cyproheptadine, a nonselective 5-HT blocker, prevented facilitation of stepping by epidural stimulation. These data demonstrate an important role of the serotonergic system in facilitating locomotion in the presence of epidural stimulation. In the presence of ketanserin, passive movements of one forelimb in a step-like manner immediately induced stepping of both hindlimbs with EMG patterns similar to those observed with ES without ketanserin. Thus, a non-5-HT-dependent spinal circuitry projecting from the cervical to the lumbar region of the spinal cord can facilitate stepping. The specific neurotransmitters responsible for this forelimb-facilitated stepping of the hindlimbs are unknown. These data suggest that a $5-\mathrm{HT}_{2 / 7}$ receptor-dependent pathway that processes hindlimb locomotor-like proprioception to facilitate hindlimb stepping can be complemented with proprioceptive afferents from the forelimbs via a non-5- $\mathrm{HT}_{2 / 7}$ receptor neurotransmitter system. Thus, different neurotransmitter receptor systems can be used to mediate the same type of sensory event, i.e., locomotor-like proprioception to facilitate the same motor task, i.e., hindlimb stepping.

\section{Introduction}

In decerebrated cats, electrical epidural stimulation (ES) of the lumbar spinal cord elicits coordinated hindlimb weight-bearing stepping with plantar foot placement (Iwahara et al., 1991; Gerasimenko et al., 2003). Although the neurotransmitter systems that mediate hindlimb spinal locomotion in response to ES are not well understood and undoubtedly involve multiple neurotransmitter systems, it is clear that serotonergic receptors play an important role. Quipazine [nonselective agonist of 5-hydroxytryptamine (serotonin) (5-HT) receptors] can be administered at a dose that facilitates, but does not induce, locomotor activity in adult spinal cats (Barbeau and Rossignol, 1991). In neonatal rats, brainstem-evoked fictive locomotion can be blocked by $5-\mathrm{HT}_{2}$ and $5-\mathrm{HT}_{7}$ receptor antagonists (Jordan and Schmidt, 2002). We have shown that appropriate doses of quipazine also can enable, while not directly inducing, stepping in complete chronic spinal adult rats during ES when the hindlimbs are placed on a moving treadmill belt (Gerasimenko et al., 2007). These studies demonstrate that step-enabling strategies can bring

\footnotetext{
Received Dec. 19, 2008; revised Feb. 11, 2009; accepted March 31, 2009.

This study was supported by National Institute of Neurological Disorders and Stroke Grant NS16333, U.S. Civilian Research and Development Foundation Grant RUB1-2872-ST-07, Russian Foundation for Basic Research Grants 07-04-00526 and 08-04-00688, the Christopher and Dana Reeve Foundation, and the California Roman Reed Bill. Correspondence should be addressed to Dr. V. Reggie Edgerton, Department of Physiological Science, University of California, Los Angeles, 621 Charles E. Young Drive LS 1804, Los Angeles, CA 90095-1527. E-mail: vre@ucla.edu. DOI:10.1523/JNEUROSCI.6058-08.2009

Copyright (C) 2009 Society for Neuroscience $\quad 0270-6474 / 09 / 295681-09 \$ 15.00 / 0$
}

the spinal cord to a physiological state at which the motor circuits can process the sensory information in a manner sufficient to generate coordinated, weight-bearing stepping (Edgerton et al., 2008).

Given previous reports of the role of 5-HT receptors in several types of models of locomotion such as fictive locomotion in the neonatal spinal cord and actual stepping after a complete spinal cord transection in the adult and in acute decerebrate preparations (for review, see Hochman et al., 2001), we hypothesized that a $5-\mathrm{HT}_{2 / 7}$ antagonist would depress locomotion in the presence of ES in decerebrated cats. We observed that ketanserin (K; an antagonist of 5- $\mathrm{HT}_{2 / 7}$ receptors) depressed and cyproheptadine (a nonselective 5-HT antagonist) blocked hindlimb stepping in decerebrated cats receiving ES. In the presence of ketanserin or cyproheptadine plus ES, however, robust stepping could be facilitated by passively moving one forelimb in a step-like manner. These observations demonstrate that ES of the dorsum of the lumbosacral spinal cord facilitates hindlimb stepping via a $5-\mathrm{HT}_{2 / 7}$ receptor-dependent pathway in the presence of proprioceptive input from the hindlimbs, whereas ES at the same lumbosacral spinal cord site can facilitate hindlimb stepping via a non-5- $\mathrm{HT}_{2 / 7}$ receptor-mediated pathway when step-like proprioceptive input is provided from the forelimbs.

\section{Materials and Methods}

All animal procedures were conducted according to the European Community Council Directive (24 November 1986, 86/609/EEC) using pro- 



EMG burst amplitude

EMG burst duration
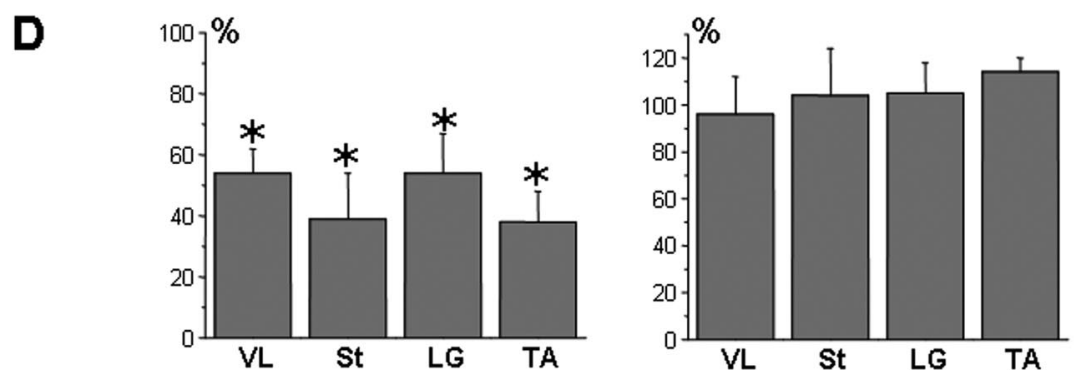

Figure 1. Effects of ketanserin (K) on the stepping pattern induced by ES in decerebrated cats. EMG recordings from the left VL (LVL), left St (LSt), left $L G(L L G)$, left TA (LTA), right $L G$ (RLG), and right TA (RTA) muscles during stepping at a belt speed of $0.3-0.4 \mathrm{~m} / \mathrm{s}$ induced by ES alone $(\boldsymbol{A})$ or by ES plus K administration (ES $+K)(\boldsymbol{B})$. Note that the administration of $K$ markedly reduces the EMG bursting in all muscles. C, Videographic recordings of one step cycle on the treadmill at a belt speed of $0.3-0.4 \mathrm{~m} / \mathrm{s}$ during ES and ES +K are shown. Note the robust step kinematics induced by ES, whereas little movement of the limb is observed after K administration. $D$, Change of mean amplitude of the EMG bursts and the mean EMG burst duration in VL, St, LG, and TA of the left hindlimb during $E S$ after administration of $K$ relative to that seen for $E S$ alone are shown. ${ }^{*} p<0.05$, significant difference between ES alone and ES $+K$.

tocols approved by the Ethics Committee of the Pavlov Institute of Physiology, St. Petersburg, Russia.

Animal preparation. These acute experiments were performed on six adult cats (2-3 kg body weight). Animals were anesthetized for surgery with a mixture of xylazine $(1 \mathrm{mg} / \mathrm{kg})$ and ketamine $(40 \mathrm{mg} / \mathrm{kg})$ administered intramuscularly. After tracheal intubation and ligation of the carotid arteries, a precollicular postmammillary decerebration was performed as described previously (Gerasimenko et al., 2003; Musienko et al., 2007). Anesthesia was discontinued after the decerebration, and the experiments were initiated 2-3 h later. A partial laminectomy was performed from L2 to L7: the cat then was fixed in a stereotaxic frame and suspended in a harness with all four limbs pendant. The exposed lumbosacral spinal cord was covered with warm paraffin oil maintained at $35-37^{\circ} \mathrm{C}$. Blood pressure was monitored via a carotid artery cannula and maintained above $80 \mathrm{mmHg}$. Rectal temperature was monitored and maintained at $\sim 38^{\circ} \mathrm{C}$ by heat irradiation.

Epidural stimulation of the spinal cord. A silver ball electrode $(2 \mathrm{~mm}$ diameter) placed on the middle, dorsal surface of the spinal cord was used for ES (monopolar) at spinal segments L4-L5. A Teflon-coated stainless steel wire (AS632; Cooner Wire) was inserted subcutaneously in the mid-back region ( $1 \mathrm{~cm}$ of Teflon removed at the distal end) and served as an indifferent ground electrode. Continuous stimulation at a frequency of $5 \mathrm{~Hz}$, a pulse duration of $0.5 \mathrm{~ms}$, and an intensity of 20-100 $\mu \mathrm{A}$ was used to induce and facilitate stepping movements in the hindlimbs; the belt of a motorized treadmill was moving at a rate of $0.3 \mathrm{~m} / \mathrm{s}$, i.e., the speed at which the most consistent stepping generally occurred, as described previously (Gerasimenko et al., 2003, 2005). ES-evoked responses (at $0.3 \mathrm{~Hz}$ stimulation) were recorded in selected hindlimb muscles during standing (see below).

EMG recording and movement analysis. Two Teflon-coated, stainless steel wires were inserted bilaterally into the mid-belly of the following muscles: vastus lateralis $(\mathrm{VL})$, semitendinosus (St), lateral gastrocnemius (LG), and tibialis anterior (TA) in the hindlimbs and the triceps brachi (TB) and biceps brachi (BB) in the forelimbs. Bipolar intramuscular EMG electrodes were formed and secured into the mid-belly of each muscle as described previously (Roy et al., 1991). The proximal ends of the wires were soldered to an amplifier connector (AM System; model 1700). The EMG signals were differentially amplified (bandwidth of 30 $\mathrm{Hz}$ to $10 \mathrm{kHz}$ ) and digitized at $2 \mathrm{kHz}$ using a National Instrument analog/ digital board and analyzed with computer programs developed with the LabView package. The EMG signals were rectified and integrated as calculated by Gilbert transformation (Bendat and Piersol, 2000). Cycle period was calculated as the time between the onset of an EMG burst and the onset of the following EMG burst from the same muscle. EMG burst durations were calculated as the time between the onset and the end of each burst. Peak EMG amplitude was determined from each rectified burst. During continuous ES at $5 \mathrm{~Hz}$, the evoked responses within the EMG burst to each epidural stimulus were categorized as a short latency potential (SLP) or a late latency complex (LLC). We calculated the integrated area for the SLP (from the onset of the stimulus to $25 \mathrm{~ms}$ ) and for the LLC (from 26 to $196 \mathrm{~ms}$ ) (Gerasimenko et al., 2005). ES at $0.3 \mathrm{~Hz}$ and with a suprathreshold intensity was delivered during standing: 10 ES- 



Figure 2. EMG activity recorded in the hindlimb muscles of a decerebrated cat during stepping with ES (at $5 \mathrm{~Hz}$ ) and after the cessation of ES. Stepping data are recorded during ES alone $(\boldsymbol{A}), \mathrm{ES}$ in the presence of $\mathrm{K}(\boldsymbol{B})$, and $\mathrm{ES}$ in the presence of K plus passive forelimb (FL) movements (C). Note that the administration of $K$ decreases the EMG bursting activity during and after the cessation of ES compared with the ES alone condition (compare $\boldsymbol{B}$ with $\boldsymbol{A}$ ). Passive FL movements under the $E S+K$ condition restored the EMG activity to that observed with $E S$ alone (compare $\mathbf{C}$ with $\boldsymbol{A}$ ). Abbreviations, same as in Figure 1.

evoked responses were averaged, and the maximal amplitude of the averaged responses was determined.

Movements of the hindlimbs were monitored using a digital video camera. Reflective markers placed on the femoral head, lateral condyle of the femur, lateral malleolus, and the fifth metatarsal were used to reconstruct the kinematics of the stepping movements. Based on the video recordings, an example of the changes in limb position between preketanserin and postketanserin administration are presented in Figure $1 C$.

Repetitive passive movements of one forelimb were induced manually at a rate of $\sim 1 \mathrm{~Hz}$. We either imposed weight bearing of the forelimb on the treadmill belt or moved the forelimb in a step-like manner without the forelimb having contact with the treadmill belt.

Drug injections. Ketanserin tartrate (Sigma), a blocker of 5- $\mathrm{HT}_{2 / 7}$ receptors, was dissolved in sterile water and administered intravenously $(0.1 \mathrm{mg} / \mathrm{kg})$ through a catheter in the radial vein (Bosco et al., 2003). The effects of ketanserin on stepping performance facilitated by ES were eval-

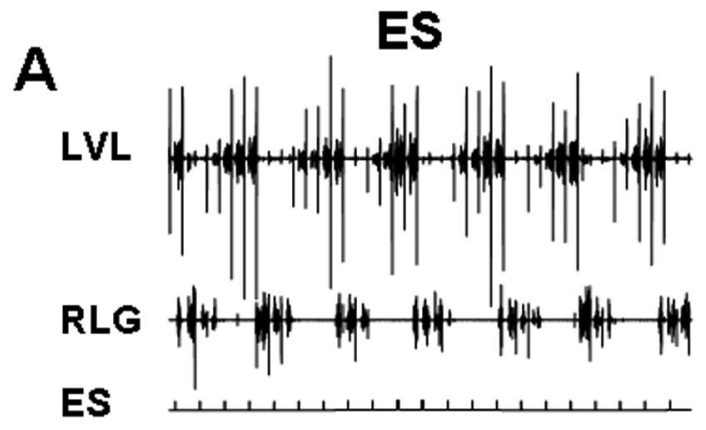

ES+cyproheptadine

LVL

RLG

ES


Figure 3. Effects of cyproheptadine on the EMG activity induced by ES. EMG activity in the LVL and RLG during ES alone $(\boldsymbol{A})$, ES in the presence of cyproheptadine $(\boldsymbol{B})$, and ES in the presence of cyproheptadine plus forelimb movements ( $\boldsymbol{C}$ are shown. Note the inhibition of EMG activity by cyproheptadine administration and the recovery of activity with $\mathrm{FL}$ movement. Abbreviations, same as in Figures 1 and 2.

uated between 30 and $180 \mathrm{~min}$ after injection. The maximal effect of ketanserin was observed $\sim 30$ min after drug administration, and the effect lasted $2-3 \mathrm{~h}$, at which time the locomotor performance recovered to preketanserin levels. The same stimulation parameters were used before and after ketanserin administration. In addition, the effects of cyproheptadine dissolved in sterile water [3-5 mg/ $\mathrm{kg}$, i.v., as described by Sastry and Sinclair (1976)] on stepping performance were determined in two cats.

Statistical analyses. The values are reported as mean \pm SEM. Statistically significant differences in each of the variables studied during ES before and after ketanserin or cyproheptadine administration and with and without forelimb passive movement were determined using a oneway repeated-measures ANOVA. Values that were not normally distributed were analyzed using the nonparametric T-White rank test. Paired Student's $t$ tests and nonparametric Wilcoxon tests were used to assess statistical differences between EMG characteristics during ES and ES plus ketanserin administration as well as between ES plus ketanserin and ES plus ketanserin plus forelimb movement. Significance was determined at the $p<0.05$ level.

\section{Results}

Characteristics of locomotor activity in decerebrated cats during $E S$ and ketanserin or cyproheptadine administration ES applied at L4-L5 ( $5 \mathrm{~Hz}, 80-100 \mu \mathrm{A})$ in the absence of ketanserin routinely elicited coordinated hindlimb stepping on the 
moving treadmill belt with robust alternating flexor-extensor EMG bursts (Fig. $1 A)$ and weight-bearing stepping with plantar foot placement (Fig. $1 C$; see supplemental Video 1, available at www. jneurosci.org as supplemental material). After ketanserin administration, the EMG activity was depressed (Fig. $1 B$ ), and only weak rhythmic movements without plantar foot placement were observed (Fig. $1 C)$. The mean EMG amplitude in the hindlimb muscles after ketanserin administration was $\sim 50 \%$ of the values obtained during ES alone, whereas the EMG burst duration was not affected significantly (Fig. 1D).

Consistent weight-bearing stepping on the moving treadmill belt was observed in all decerebrated cats under ES. In the example shown in Figure $2 \mathrm{~A}$, stepping continued for $\sim 12 \mathrm{~s}$ when the treadmill belt continued to move after ES was stopped. The more typical persistence of stepping lasted $\sim 5 \mathrm{~s}$. During this period, the amplitude of the EMG bursts progressively decreased until there were no EMG bursts generated. An obvious difference between the EMG bursts with and without ES was the absence of high amplitude-induced potentials without ES. Based on visual inspection, the quality of the stepping consistently appeared to be superior immediately after the cessation of ES. In the presence of ketanserin, the EMG activity during ES was depressed (Fig. 2B): after the cessation of ES, the EMG remained depressed, and the muscles became quiescent very rapidly. Administration of cyproheptadine completely blocked ESfacilitated stepping (Fig. 3A,B).

\section{Effects of passive forelimb movements on the locomotor activity during ES and ketanserin or cyproheptadine administration in decerebrated cats}

During subthreshold ES for eliciting hindlimb stepping, the manual movement of one forelimb in a step-like manner initiated stepping in the hindlimbs of the decerebrated cats. In the absence of ES, however, manually moving the forelimb did not initiate hindlimb stepping movements. In addition, when robust hindlimb stepping was induced by suprathreshold ES, passive forelimb movements did not result in any observable modulation in hindlimb stepping. When the EMG activity in the hindlimb muscles elicited by ES was depressed by ketanserin administration, the movement of one forelimb in a step-like manner immediately initiated hindlimb stepping (see supplemental Video 2, available at www.jneurosci.org as supplemental material). The EMG activity during hindlimb stepping with passive forelimb movement was similar to the EMG patterns observed during ES without ketanserin and no forelimb passive movement (compare Fig. $2 A, C$ ).

As occurred in the presence of ketanserin, the hindlimbs recovered stepping movements after cyproheptadine administration when one forelimb was moved passively in a step-like manner (Fig. 3C).

We categorized the evoked EMG responses to individual epi- dural stimuli within the EMG burst during stepping as having an SLP component and an LLC (see expanded EMG activity at the bottom of Fig. 4). The latency of the SLP was $\sim 10 \mathrm{~ms}$ (Fig. 4) and most likely corresponds to the middle response (MR) observed in the hindlimb muscles in intact (Gerasimenko et al., 2006) as well as spinal (Lavrov et al., 2006) rats in response to ES. The LLC seems to correspond to the late response in rats, although the latency is longer in the cat than rat.

The superposition of four EMG responses to epidural stimuli $(5 \mathrm{~Hz})$ within a single VL EMG burst with ES alone, ES plus ketanserin, and ES plus ketanserin plus passive forelimb movements are shown in Figure 5A. Both the SLP and LLC responses (as illustrated in Fig. 4) were quantified based on the integral of each rectified EMG response during the burst associated with a step. These data were calculated for the VL, St, LG, and TA during stepping with ES alone, ES with ketanserin, ES plus ketanserin plus forelimb movement and during ES after recovery from ketanserin (Fig. $5 B$, values are expressed as a percentage of ES alone before drug administration). In all muscles of all animals, ketanserin administration $(0.1 \mathrm{mg} / \mathrm{kg}$, i.v. $)$ significantly decreased the integrated area of both the SLP (to $\sim 25 \%$ of ES alone) and LLC (to $\sim 40 \%$ of ES alone) responses induced by ES during hindlimb stepping (Fig. 5B). When the forelimbs were moved passively during hindlimb stepping, these values were restored to $\sim 50-$ $90 \%$ of the values with ES alone. The mean differences between ES plus ketanserin and ES plus ketanserin plus passive forelimb movement were significant in the VL for both the SLP (22 and $59 \%$, respectively) and the LLC ( 24 and $88 \%$, respectively) as well as in the TA for the LLC (56 and 105\%, respectively). 
A

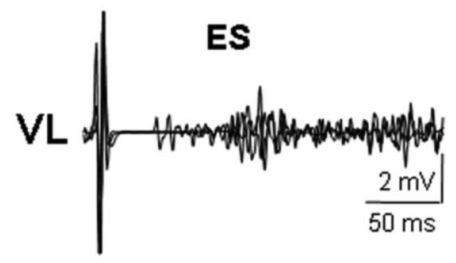

ES+K
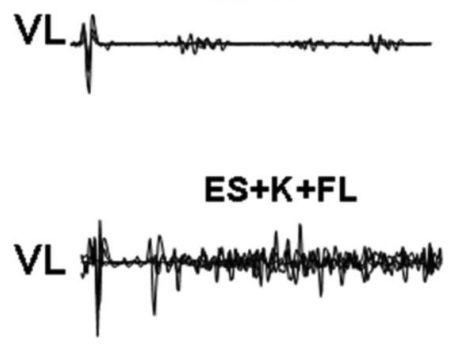

B

SLP
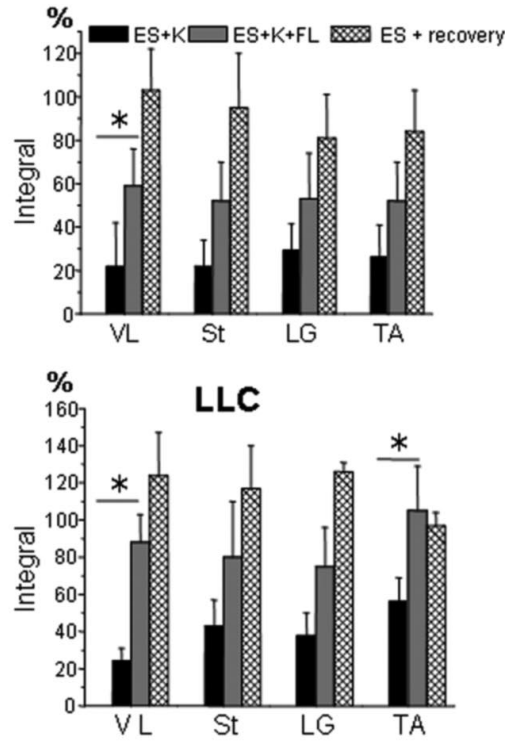

C

SLP
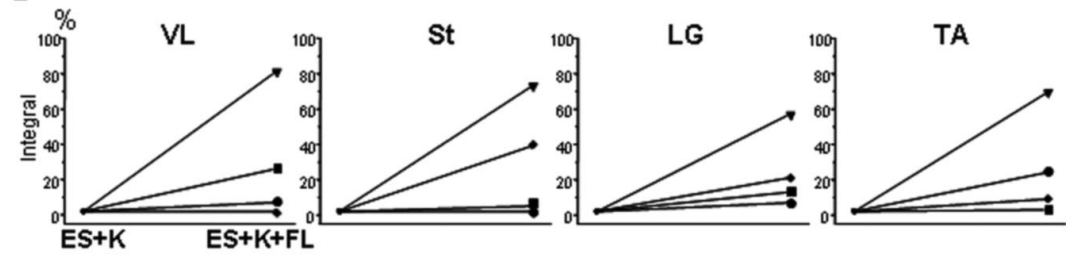

LLC
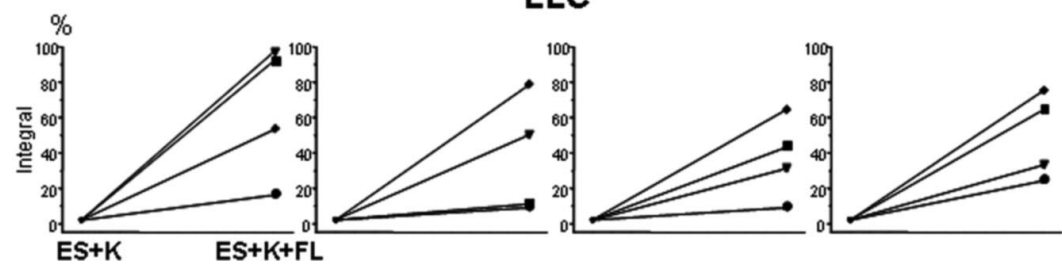

Figure 5. Superposition of four responses within a single VL EMG burst during ES alone, ES $+K$, and $E S+K+F L$ are shown in $A$. The mean integral (expressed as a percentage of pre-K administration) of the SLP and LLC within the VL, St, LG, and TA EMG bursts during stepping with ES +K, ES + K + FL as well as during ES $\sim 2-2.5 \mathrm{~h}$ after K administration (ES recovery) are shown in $B$. Note the significantly smaller responses under $E S+K$, the partial recovery with $F L$ movements, and the restoration of the responses to or above pre-K values after recovery. Significant difference between $E S+K$ and $E S+K+F L$ at ${ }^{*} p<0.05$. In all cases, ES recovery is significantly different from ES $+K$. In $C$, the percentage change in the integral of the SLP and LLC for each of four animals. All data are collected from muscles on the same side. Each symbol represents the same animal for each of the four muscles. Abbreviations, same as in Figures 1 and 2.

Although the other mean comparisons were not significant, each muscle in all animals showed an elevated EMG response when the forelimb was moved passively compared with ES plus ketanserin (Fig. 5C). By $\sim 2 \mathrm{~h}$ after drug administration, the integrals of both the SLP and LLC responses were at or above the preketanserin values.

Based on the EMG patterns, the quality of the hindlimb stepping during ES in the presence of ketanserin was more robust when the forelimb was stepping with weight bearing than air stepping (Fig. 6, compare $A, B$ ). Whereas the cycle period was similar during both types of stepping (Fig. $6 \mathrm{C}$ ), the EMG burst duration of the VL increased, and the integral of the EMG burst of both the VL and LG was higher when weight-bearing stepping was imposed on one forelimb compared with air stepping.

ES-evoked responses in the hindlimb muscles during standing before and after ketanserin administration In decerebrated cats, we recorded evoked responses in the hindlimb muscles to ES (L4-L5, $0.3 \mathrm{~Hz}$ ) at a suprathreshold level during standing. The same parameters of ES were used after ketanserin administration. ES-evoked potentials in the hindlimb muscles had a latency of 12-15 ms (Fig. 7). Compared with ES alone, ketanserin resulted in a depression $(\sim 50 \%)$ of the ESevoked potentials in each of the hindlimb muscles studied in all cats during standing.

\section{Discussion}

Epidural stimulation-facilitated hindlimb stepping in decerebrated cats is mediated in large part by $5-\mathrm{HT}_{2 / 7}$ receptors

In chronic spinal animals, stepping can be induced or facilitated by quipazine (a nonselective agonist of 5 - $\mathrm{HT}$ receptors) (Barbeau and Rossignol, 1990; Antri et al., 2002; Fong et al., 2005). ES of the lumbar spinal cord also facilitates locomotorlike hindlimb movements in decerebrated (Gerasimenko et al., 2005) and spinal (Gerasimenko et al., 2003) cats as well as in chronic spinal adult rats (Ichiyama et al., 2005). In the presence of quipazine, ES significantly facilitates stepping in chronic spinal 
rats (Gerasimenko et al., 2007). These observations, however, provide no insight into which components of the spinal circuitry are responsible for the motor patterns. Based on the ketanserin effects, the present data show that hindlimb stepping can be facilitated or induced via $5-\mathrm{HT}_{2 / 7}$ receptors when the lumbosacral spinal cord of a decerebrated cat is stimulated epidurally at L4-L5. In some cats, alternating activity of the hindlimbs at the time of the maximum effect of ketanserin was suppressed, but not completely blocked, in response to ES. Thus, the dosage of ketanserin used in the present study is consistent with there being an effective, but incomplete, block of $5-\mathrm{HT}_{2 / 7}$ receptors, i.e., the administration of this dosage suppressed locomotor performance during ES in all cats tested. Under the same stimulating conditions, cyproheptadine administration completely eliminated the locomotion induced by ES, demonstrating the importance of the serotonergic system in the regulation of locomotion induced by ES. The greater effect of cyproheptadine on locomotion, however, cannot be assumed to be attributable to a more complete block of only 5-HT receptors because of its nonspecificity. For example, cyproheptadine has the potential to block muscarinic receptors, having a similar high affinity for both serotonin and muscarinic receptors (Eltze et al., 1989).

The ES-evoked potentials had the properties of a monosynaptic response as they were inhibited during muscle vibration and double epidural stimulation (see supplemental Fig. 1, available at www.jneurosci.org as supplemental material). Thus, these potentials probably are monosynaptic responses to ES and may correspond to the MR responses recorded in hindlimb muscles of intact (Gerasimenko et al., 2006) and spinal (Lavrov et al., 2006) rats to ES during bipedal standing. Consequently, these results suggest that ketanserin depressed the ES-evoked monosynaptic responses. It is interesting, however, that during hindlimb stepping with ES at $5 \mathrm{~Hz}$ and in the presence of ketanserin, the SLP was inhibited more strongly than when evoked at a rate of $0.3 \mathrm{~Hz}$ in the presence of ketanserin during standing. Passive forelimb movements, however, facilitated the SLP to values obtained during standing. The amplitude of evoked potentials in the hindlimb muscles induced by ES $(0.3 \mathrm{~Hz})$ during standing decreased $\sim 50 \%$ after ketanserin administration.

Passive step-like forelimb movements can induce hindlimb stepping on a moving treadmill belt in the presence of serotonergic blockers and ES

Although ketanserin suppressed ES-facilitated hindlimb locomotion, passive step-like movements of one forelimb immediately induced stepping of the hindlimbs on a moving tread-
B

ES+K+FL weight - bearing stepping

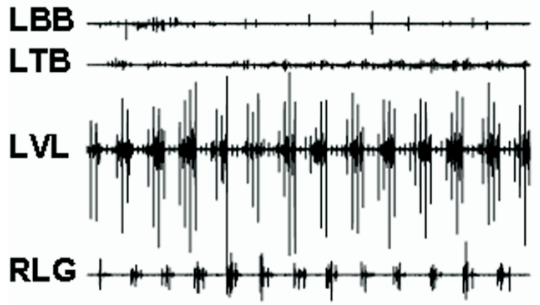

ES
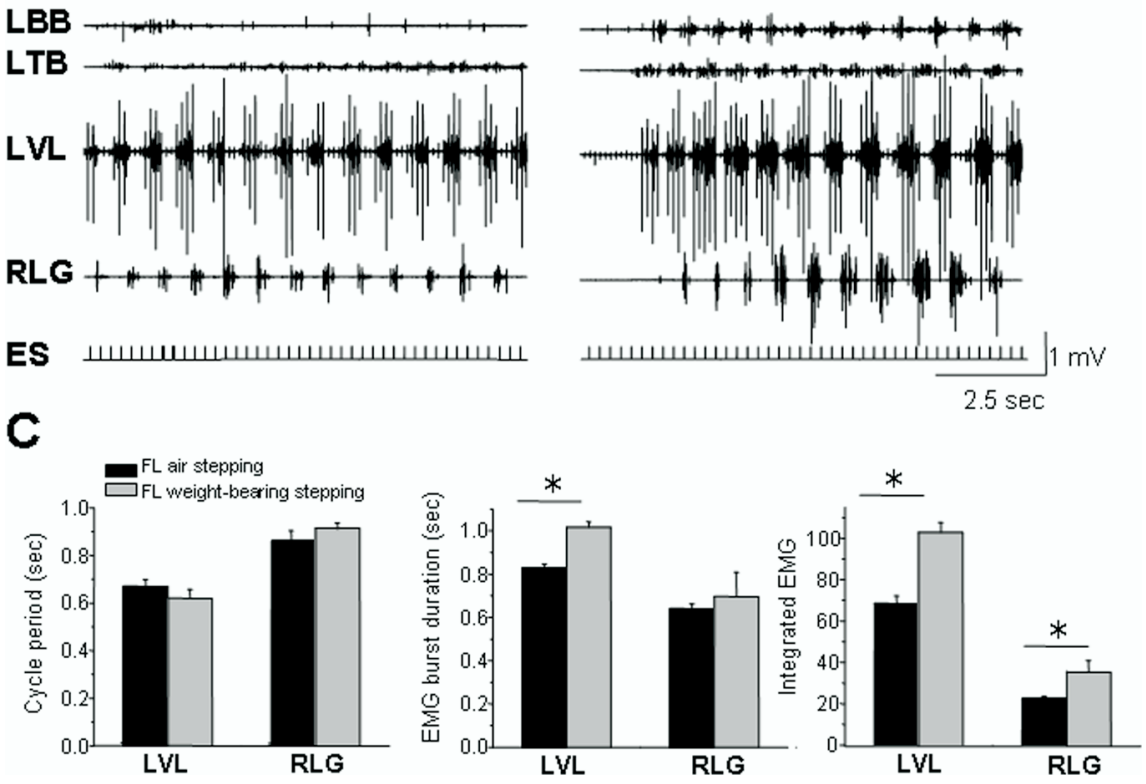

Figure 6. Effect of quality of forelimb movements in a decerebrate cat on stepping performance of the hindlimbs during ES in the presence of $K$. EMG recordings in the LBB, LTB, LVL, and RLG during ES-induced stepping in the presence of $K$ plus air stepping $(\boldsymbol{A})$ or weight-bearing stepping on a treadmill $(\boldsymbol{B})$. The mean cycle period, mean EMG burst duration, and mean integrated area of the rectified EMG burst of the LVL and RLG during air stepping or weight-bearing stepping induced by ES and in presence of $K$ are shown in $\boldsymbol{C}$. Note the more robust EMG bursts in both the forelimb and hindlimb muscles during weight-bearing than during air stepping. Significantly different at ${ }^{*} p<0.05$. Abbreviations, same as in Figures 1 and 2 .

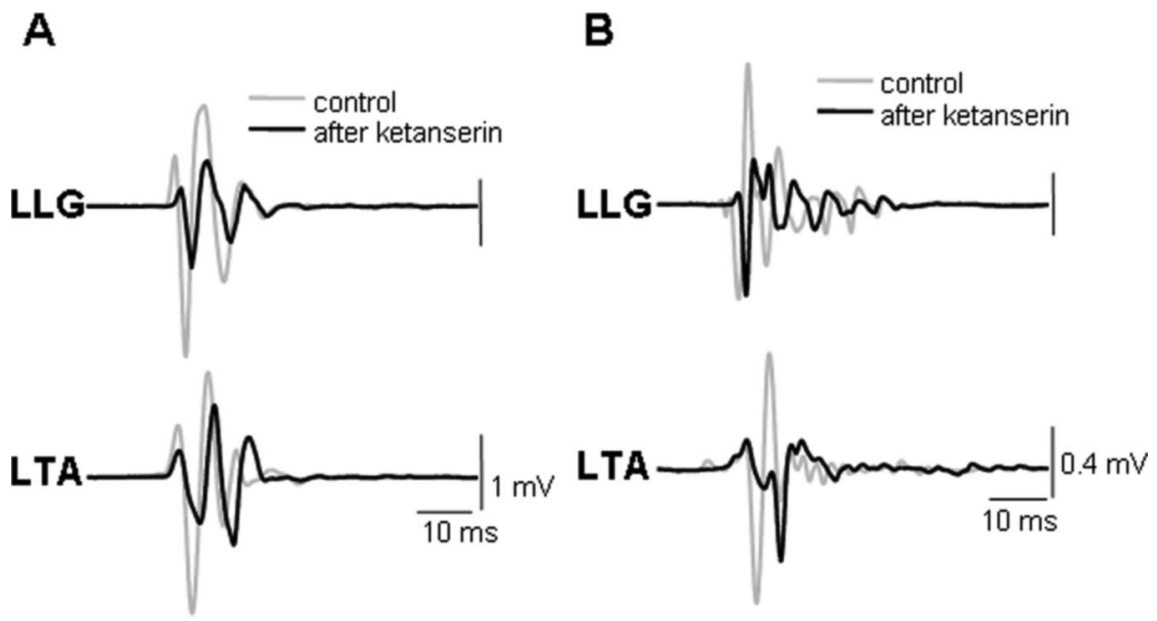

Figure 7. Averaged $(n=10)$ SLPs from the LLG and LTA in response to ES $(0.3 \mathrm{~Hz})$ during standing in two decerebrated cats $(\boldsymbol{A}$, $B$ ) are shown. The gray traces show the potentials before and the black traces after ketanserin administration. Note the lower $(\sim 50 \%)$ amplitudes after rather than before ketanserin administration. Abbreviations, same as in Figures 1 and 2.

mill belt in the presence of ES. Although the $5-\mathrm{HT}_{2 / 7}$ receptor ketanserin blocked or partially depressed locomotor activity, forelimb passive movements overcame this block. Cyproheptadine, a nonselective 5-HT antagonist, completely blocked stepping, suggesting that other receptors are important in addition to $5-\mathrm{HT}_{2 / 7}$ in mediating the ES-facilitated stepping. As noted previously, given the similarly high affinity of cyproheptadine to both serotonergic and muscarinic receptors (Eltze et al., 1989), the observed complete block could be attributed to muscarinic as well as to 5-HT receptors other than $5-\mathrm{HT}_{2 / 7}$. However, even in the presence of cyproheptadine that pro- 


\section{Ketanserin Block at the Motoneuron and/or Interneuron Level}

A

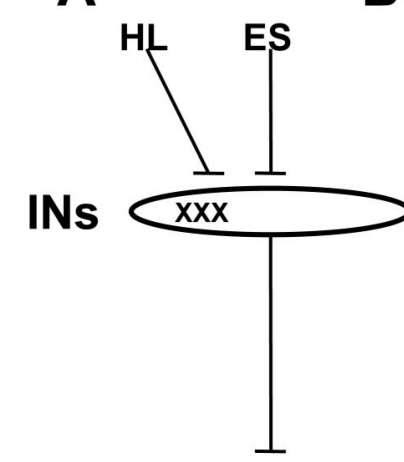

MNs

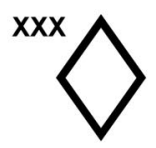

Locom

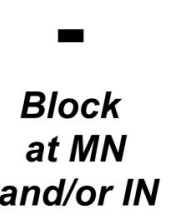

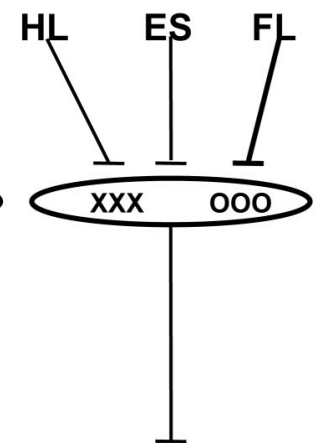
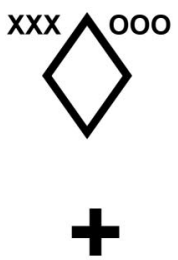

\section{Block at $M N$, but FL activated via Non - $5-H T_{2 / 7} R$}

\section{$\mathrm{XXX}-5-H T_{2 / 7} R ; 000-N o n-5-H T_{2 / 7} R$}

Figure 8. Hypothetical blocks at the motoneurons and/or at the interneurons are illustrated. Robust hindlimb locomotion (Locom) occurs during ES and when step-like sensory information is projected from the hindlimbs $(\mathrm{HL})$ when they are placed on a moving treadmill belt. Ketanserin blocks this effect $(-)(\boldsymbol{A})$. When one forelimb is moved in a step-like manner in the presence of a ketanserin block, the hindlimbs begin stepping almost immediately $(+)(\boldsymbol{B})$. These results together demonstrate that those pathways through which the hindlimb sensory information and $\mathrm{ES}$ activate stepping are mediated via $5-\mathrm{HT}_{2 / 7}$ receptors ( $\mathrm{X}$ 's), but sensory information from the forelimb can activate pathways that do not depend on $5-\mathrm{HT}_{2 / 7}$ receptors $\left(0^{\prime} s\right)$. A block at the level of interneurons may occur by activating different populations of interneurons or via the same population of interneurons having both $5-\mathrm{HT}_{2 / 7}$ and non-5- $\mathrm{HT}_{2 / 7}$ receptors $(\boldsymbol{B})$. Based on our current understanding of motor unit recruitment, the ketanserin block at the level of the motoneuron would probably occur as a result of the same motoneurons having both 5- $\mathrm{HT}_{2 / 7}$ and non-5- $\mathrm{HT}_{2 / 7}$ receptors. - , Suppression of hindlimb locomotion; +, facilitation of hindlimb locomotion; $\mathrm{MN}$, motoneuron; IN, interneuron.

duced a complete block of ES-induced stepping, passive movement of one forelimb was still able to induce robust hindlimb stepping. Figure 8 shows a scheme of activation of neurons via 5-HT-dependent and 5-HT-independent neuronal "pools" that could mediate locomotor performance during ES. Hypothetically, the site of action of the pharmacological block could range from sites among motoneurons or populations of interneurons or some combination of the two. The scenario in Figure 8 demonstrates that when one forelimb is moved in a step-like manner when there is a ketanserin block, the hindlimbs begin stepping almost immediately. These results demonstrate that those pathways through which the hindlimb sensory information and the ES activates stepping is mediated via 5- $\mathrm{HT}_{2 / 7}$ receptors, but sensory information from the forelimb can activate pathways that do not depend on $5-\mathrm{HT}_{2 / 7}$ receptors. A block at the level of the interneurons may

occur via different populations of interneurons having either $5-\mathrm{HT}_{2 / 7}$ or non-5- $\mathrm{HT}_{2 / 7}$ receptors or the same population of interneurons having both $5-\mathrm{HT}_{2 / 7}$ and non-5- $\mathrm{HT}_{2 / 7}$ receptors. Based on our current understanding of motoneuron recruitment, it is unlikely that different populations of motoneurons are recruited to accomplish the same motor task. Thus, if the ketanserin block is at the level of the motoneuron, this probably occurs as a result of the same motoneurons having both 5- $\mathrm{HT}_{2 / 7}$ and non-5- $\mathrm{HT}_{2 / 7}$ receptors, one serving hindlimbderived proprioception and the other forelimb-derived proprioception.

The specific neurotransmitter systems responsible for inducing this forelimb-facilitated stepping of the hindlimbs are unknown. Cholinergic systems could contribute to this interlimb coordination since blocking cholinergic transmission by atropine (an antagonist of muscarinic receptors) disrupts the rhythmic coupling activity between the cervical and lumbar locomotor networks in the neonatal-isolated spinal cord (Cowley and Schmidt, 2000). Other neurotransmitter systems supporting interlimb coordination could be noradrenergic (Bergmans et al., 1973), glutamatergic (Douglas et al., 1993; Wallis and $\mathrm{Wu}, 1993$ ), GABAergic, or glycinergic (Hinckley et al., 2005).

As previously demonstrated by Miller et al. (1975), Yamaguchi (1986), and Juvin et al. (2005), we show that there are neural circuits in the cervical spinal cord that can initiate hindlimb stepping. The novelty of the present observations is that we show a forelimb-to-hindlimb pathway in which locomotor-like proprioception from the forelimbs facilitates (in conjunction with ES) hindlimb locomotion that functions mainly independently of $5-\mathrm{HT}_{2 / 7}$ receptors, whereas the proprioception derived from the hindlimbs in this same experimental situation is mostly dependent on $5-\mathrm{HT}_{2 / 7}$ receptors.

Given that the present experiments were performed in an acute preparation of decerebrated cats and that reflex-induced interconnections between the forelimb and hindlimb seem to be enhanced after a chronic spinal cord injury (Calancie et al., 2002), the forelimb proprioception-facilitating effects observed here may become even more prominent after spinal cord injury. Dietz et al. (1999) suggested that human subjects with a severe cervical spinal cord lesion demonstrate more rhythmical EMG in the lower limbs while suspended with partial weight support on a treadmill than do individuals with a mid-thoracic lesion.

\section{Propriospinal coupling between cervical and lumbar locomotor networks}

In quadrupedal animals, there are clear functional interconnections between the locomotor networks for the forelimbs and for the hindlimbs, providing interlimb coupling (Cazalets and Bertrand, 2000; Ballion et al., 2001; Juvin et al., 2005). Shik and Orlovsky (1965) reported that when the two forelimbs of intact dogs are stepping on a treadmill belt, the suspended hindlimbs perform step-like movements in the air, but when the hindlimbs are stepping on a treadmill, the suspended forelimbs do not move. Thus, their conclusion was that the influence of the forelimbs on the hindlimbs is stronger than the reverse. Akay et al. (2006) also observed asymmetry in the descending and ascending linkages between neuronal systems controlling stepping in the forelimbs and hindlimbs in decerebrated cats. We did not observe locomotor-like activity of the forelimbs, however, when ES (at L4-L5) was used to elicit weight-bearing hindlimb stepping in decerebrated cats. Pas- 
sive movement of the forelimbs also had no effect on the stepping performance of the hindlimbs during ES in the absence of a serotonergic blocker.

Interlimb coordination is provided by both intraspinal and afferent processing mechanisms. Miller et al. (1975) reported that after complete deafferentation of both hindlimbs, quadrupedal stepping similar to that observed predeafferentation can be generated in decerebrated cats. This observation demonstrates the importance of the propriospinal circuitry in forelimb-hindlimb coordination. Long propriospinal neurons have been suggested to play an important role in the functional coupling of the cervical and lumbar locomotor networks, providing forelimb-hindlimb coordination (Yamaguchi, 1986). Such neurons have been described in both the rat (Menétrey et al., 1985) and cat (Skinner et al., 1980; Verburgh et al., 1989). Faganel and Dimitrijevic (1982) characterized the propriospinal interneuron system in clinically complete spinal cord injured patients. They demonstrated an increase in the amplitude of the Achilles tendon reflex during conditioning noxious electrical stimulation of the thoracic, lumbar, or sacral dermatomes, suggesting that this effect is mediated by the propriospinal system.

Propriospinal reflexes at rest and during locomotion have been studied in detail (Lloyd, 1942; Schomburg et al., 1978; Skinner et al., 1980), demonstrating their importance in interlimb coordination. During locomotion, the long spinal reflexes induced by electrical stimulation of cutaneous nerves were modulated in a step phase-dependent manner (Miller et al., 1977). Shik (1997) proposed the concept of initiation of locomotion through the propriospinal system. He suggested that neurons of the dorsal and ventral part of the lateral funiculus form a propriospinal system that can facilitate stepping.

Although the present results are consistent with the influence of forelimb proprioception on hindlimb stepping, they are unique in demonstrating that, unlike the hindlimb facilitation, the forelimb effect is mediated through non-5- $\mathrm{HT}_{2 / 7}$ receptor-dependent pathways. Identification of the specific serotonergic and other relevant neurotransmitter systems that enable hindlimb stepping may be useful in formulating pharmacological strategies to regain locomotor function after a severe spinal cord injury.

\section{References}

Akay T, McVea DA, Tachibana A, Pearson KG (2006) Coordination of fore and hind leg stepping in cats on a transversely-split treadmill. Exp Brain Res 175:211-222.

Antri M, Orsal D, Barthe JY (2002) Locomotor recovery in the chronic spinal rat: effects of long-term treatment with a 5-HT2 agonist. Eur J Neurosci 16:467-476.

Ballion B, Morin D, Viala D (2001) Forelimb locomotor generators and quadrupedal locomotion in the neonatal rat. Eur J Neurosci 14:1727-1738.

Barbeau H, Rossignol S (1990) The effects of serotonergic drugs on the locomotor pattern and on cutaneous reflexes of the adult chronic spinal cat. Brain Res 514:55-67.

Barbeau H, Rossignol S (1991) Initiation and modulation of locomotor pattern in the adult chronic spinal cat by noradrenergic, serotonergic and dopaminergic drugs. Brain Res 546:250-260.

Bendat J, Piersol A (2000) Random data. Analysis and measurement procedures. New York: Wiley.

Bergmans J, Miller S, Reitsma DJ (1973) Influence of L-DOPA on transmission in long ascending propriospinal pathways in the cat. Brain Res 62:155-167.

Bosco G, Rankin A, Poppele RE (2003) Modulation of dorsal spinocerebellar responses to limb movement. I. Effect of serotonin. J Neurophysiol 90:3361-3371.
Calancie B, Molano MR, Broton JG (2002) Interlimb reflexes and synaptic plasticity become evident months after human spinal cord injury. Brain 125:1150-1161.

Cazalets JR, Bertrand S (2000) Coupling between lumbar and sacral motor networks in the neonatal rat spinal cord. Eur J Neurosci 12:2993-3002.

Cowley K, Schmidt B (2000) Characterization of propriospinal coupling between the cervical and lumbar regions during rhythmic motor activity in the in vitro neonatal rat spinal cord. Soc Neurosci Abstr 26:60.2.

Dietz V, Nakazawa K, Wirz M, Erni T (1999) Level of spinal cord lesion determines locomotor activity in spinal man. Exp Brain Res 128:405-409.

Douglas JR, Noga BR, Dai X, Jordan LM (1993) The effects of intrathecal administration of excitatory amino acid agonists and antagonists on the initiation of locomotion in the adult cat. J Neurosci 13:990-1000.

Edgerton VR, Courtine G, Gerasimenko YP, Lavrov I, Ichiyama RM, Fong AJ, Cai LL, Otoshi CK, Tillakaratne NJ, Burdick JW, Roy RR (2008) Training locomotor networks. Brain Res Rev 57:241-254.

Eltze M, Lambrecht G, Mutschler E (1989) Cyproheptadine displays high affinity for muscarinic receptors but does not discriminate between receptor subtypes. Eur J Pharmacol 173:219-222.

Faganel J, Dimitrijevic MR (1982) Study of propriospinal interneuron system in man. Cutaneous exteroceptive conditioning of stretch reflexes. J Neurol Sci 56:155-172.

Fong AJ, Cai LL, Otoshi CK, Reinkensmeyer DJ, Burdick JW, Roy RR, Edgerton VR (2005) Spinal cord-transected mice learn to step in response to quipazine treatment and robotic training. J Neurosci 25:11738-11747.

Gerasimenko YP, Avelev VD, Nikitin OA, Lavrov IA (2003) Initiation of locomotor activity in spinal cats by epidural stimulation of the spinal cord. Neurosci Behav Physiol 33:247-254.

Gerasimenko YP, Lavrov IA, Bogacheva IN, Shcherbakova NA, Kucher VI, Musienko PE (2005) Formation of locomotor patterns in decerebrated cats in conditions of epidural stimulation of the spinal cord. Neurosci Behav Physiol 35:291-298.

Gerasimenko YP, Lavrov IA, Courtine G, Ichiyama RM, Dy CJ, Zhong H, Roy RR, Edgerton VR (2006) Spinal cord reflexes induced by epidural spinal cord stimulation in normal awake rats. J Neurosci Methods 57:253-263.

Gerasimenko YP, Ichiyama RM, Lavrov IA, Courtine G, Cai L, Zhong H, Roy RR, Edgerton VR (2007) Epidural spinal cord stimulation plus quipazine administration enable stepping in complete spinal adult rats. J Neurophysiol 98:2525-2536.

Hinckley C, Seebach B, Ziskind-Conhaim L (2005) Distinct roles of glycinergic and GABAergic inhibition in coordinating locomotor-like rhythms in the neonatal mouse spinal cord. Neuroscience 131:745-758.

Hochman S, Garraway SM, Machacek DW, Shay BL (2001) 5-HT receptors and the neuromodulatory control of spinal cord function. In: Motor neurobiology of the spinal cord (Cope TC, ed), pp 47-87. Boca Raton, FL: CRC.

Ichiyama RM, Gerasimenko YP, Zhong H, Roy RR, Edgerton VR (2005) Hindlimb stepping movements in complete spinal rats induced by epidural spinal cord stimulation. Neurosci Lett 383:339-344.

Iwahara T, Atsuta Y, Garcia-Rill E, Skinner RD (1991) Spinal cord stimulation - induced locomotion in the adult cat. Brain Res Bull 28:99-105.

Jordan LM, Schmidt BJ (2002) Propriospinal neurons involved in the control of locomotion: potential targets for repair strategies? Prog Brain Res 137:125-140.

Juvin L, Simmers J, Morin D (2005) Propriospinal circuitry underlying interlimb coordination in mammalian quadrupedal locomotion. J Neurosci 25:6025-6035.

Lavrov I, Gerasimenko YP, Ichiyama RM, Courtine G, Zhong H, Roy RR, Edgerton VR (2006) Plasticity of spinal cord reflexes after a complete transaction in adult rats: relationship to stepping ability. J Neurophysiol 96:1699-1710.

Lloyd D (1942) Mediation of descending long spinal reflex activity. J Neurophysiol 5:435-458.

Menétrey D, de Pommery J, Roudier F (1985) Propriospinal fibers reaching the lumbar enlargement in the rat. Neurosci Lett 58:257-261.

Miller S, Van Der Burg J, Van Der Meché F (1975) Coordination of the hindlimbs and forelimbs in different forms of locomotion in normal and decerebrate cats. Brain Res 91:217-237.

Miller S, Ruit JB, Van der Meché FG (1977) Reversal of sign of long spinal reflexes dependent on the phase of the step cycle in the high decerebrate cat. Brain Res 128:447-459.

Musienko PE, Bogacheva IN, Gerasimenko YP (2007) Significance of 
peripheral feedback in the generation of stepping movements during epidural stimulation of the spinal cord. Neurosci Behav Physiol 37:181-190.

Roy RR, Hutchison DL, Pierotti DJ, Hodgson JA, Edgerton VR (1991) EMG patterns of rat ankle extensors and flexors during treadmill locomotion and swimming. J Appl Physiol 70:2522-2529.

Sastry BS, Sinclair JG (1976) Serotonin involvement in the blockade of bulbospinal inhibition of the spinal monosynaptic reflex. Brain Res 115:427-436.

Schomburg ED, Meinck HM, Haustein J, Roesler J (1978) Functional organization of the spinal reflex pathways from forelimb afferents to hindlimb motoneurones in the cat. Brain Res 139:21-33.

Shik M (1997) Recognizing propriospinal and reticulospinal systems of initiation of stepping. Motor Control 1:310-313.
Shik M, Orlovsky G (1965) Coordination of the legs during running of the dogs. Biophysics 10:1148-1159.

Skinner RD, Adams RJ, Remmel RS (1980) Response of long descending propriospinal neurons to natural and electrical types of stimuli in cat. Brain Res 196:387-403.

Verburgh CA, Kuypers HG, Voogd J, Stevens HP (1989) Spinocerebellar neurons and propriospinal neurons in the cervical spinal cord: a fluorescent double labeling study in the rat and the cat. Exp Brain Res 75:73-82.

Wallis DI, Wu J (1993) The pharmacology of descending responses evoked by thoracic stimulation in the neonatal rat spinal cord in vitro. Naunyn Schmiedebergs Arch Pharmacol 347:643-651.

Yamaguchi T (1986) Descending pathways eliciting forelimb stepping in the lateral funiculus: experimental studies with stimulation and lesion of the cervical cord in decerebrate cats. Brain Res 379:125-136. 